University of Nebraska - Lincoln

DigitalCommons@University of Nebraska - Lincoln

Norman R. Simon Papers

Research Papers in Physics and Astronomy

9-15-1982

\title{
A Plea For Reexamining Heavy Element Opacities In Stars
}

Norman R. Simon

University of Nebraska - Lincoln, nsimon@unl.edu

Follow this and additional works at: https://digitalcommons.unl.edu/physicssimon

Simon, Norman R., "A Plea For Reexamining Heavy Element Opacities In Stars" (1982). Norman R. Simon Papers. 16.

https://digitalcommons.unl.edu/physicssimon/16

This Article is brought to you for free and open access by the Research Papers in Physics and Astronomy at DigitalCommons@University of Nebraska - Lincoln. It has been accepted for inclusion in Norman R. Simon Papers by an authorized administrator of DigitalCommons@University of Nebraska - Lincoln. 
The Astrophysical Journal, 260:L87-L90, 1982 September 15

(ㅇ 1982. The American Astronomical Society. All rights reserved. Printed in U.S.A.

\title{
A PLEA FOR REEXAMINING HEAVY ELEMENT OPACITIES IN STARS
}

\author{
NORMAN R. SimON \\ Behlen Laboratory of Physics, University of Nebraska-Lincoln \\ Received 1982 April 5; accepted 1982 May 28
}

\begin{abstract}
It is shown that increasing the opacity due to heavy elements by a factor of 2-3 leads to classical Cepheid models which reproduce observed period ratios at evolutionary masses and luminosities. Thus the mass anomalies are removed in both the double-mode and bump Cepheid regimes. The proposed increases may also serve to energize $\beta$ Cephei variables, thus solving yet another important problem in the theory of pulsating stars. We argue that opacity changes of this order are not implausible and urge further work in this important area.
\end{abstract}

Subject headings: opacities - stars: Cepheids - stars: pulsation

\section{INTRODUCTION}

The classical Population I Cepheids have played a historically crucial role in astronomy and astrophysics as extragalactic distance indicators and as a test of that cornerstone of modern astronomy, the theory of stellar evolution. In recent years, however, a substantial body of evidence has accumulated which indicates that serious gaps exist in our understanding of these important stars. At masses and luminosities mandated by stellar evolution calculations, standard models give period ratios far larger than those observed for the double-mode Cepheids $\left(0.70 \lesssim P_{1} / P_{0} \lesssim 0.71\right)$ or those inferred for the middle-period or "bump" Cepheids $\left(0.47 \lesssim P_{2} / P_{0}\right.$ $\lesssim 0.53)$. These discrepancies have been discussed in detail by Cox (1980). By contrast, in the RR Lyrae regime, current horizontal-branch models seem to be consistent with observed period ratios in the range 0.74 $<P_{1} / P_{0}<0.75$. This conclusion has been much bolstered by the recent detection of double-mode RR Lyrae variables in M15 (Cox, Hodson, and Clancy 1982) which now join with the single previous example, the field star AQ Leonis (Jerzykiewicz and Wenzel 1977).

Various measures have been proposed to decrease period ratios for the Population I Cepheids. These include mass loss, helium enrichment, adoption of the Carson opacities, and the inclusion of magnetic fields (see Cox 1980 for references and a detailed discussion). To the present, none of these proposals have received widespread acceptance. However, at evolutionary masses and luminosities, Carson opacity models have displayed some modest advantages over their Los Alamos opacity counterparts, both in somewhat reducing the bump Cepheid period-ratio discrepancy (Carson and Stothers 1976; Vemury and Stothers 1978) and in better matching observed light and velocity curves (Vemury and Stothers 1978; Simon, Lee, and Teays 1980; Davis and Simon 1981; Simon and Davis 1982).
It has been known since the work of Fricke, Stobie, and Strittmatter (1971) that changes in opacity can under certain conditions produce significant changes in the periods of pulsational models. (But see Petersen 1974 for a counterexample.) Recently, it was reported by Simon (1981) that an augmentation of the opacity by a factor of 2-2.5 in layers hotter than $10^{5} \mathrm{~K}$ would serve to remove completely the period-ratio discrepancy for both the double-mode and bump Cepheids. In the present Letter we argue that such increases might plausibly have their seat in the contribution to the opacity made by the elements heavier than helium, i.e., in astrophysical parlance, the "metals."

The fraction of total opacity contributed by heavy elements at a given point in a model may be estimated by comparing opacities from a mixture with normal Population I metal abundance $(Z=0.02-0.03)$ with those from a similar mixture but with small $Z$, e.g., King IVa versus King Ia from the tables of Cox and Tabor (1976). This comparison turns up heavy element contributions which reach $40 \%$ for $5 \times 10^{5} \lesssim T \lesssim 10^{6} \mathrm{~K}$ and densities appropriate to Cepheid envelopes. Similar comparisons made with the formula of Stellingwerf (1975) give similar results. That this should be so is not surprising since the above regime is within the stated range of validity of the Stellingwerf formula.

\section{LINEAR NONADIABATIC CALCULATIONS}

For computational simplicity we have used the Stellingwerf (1975) formula to test the effect on period ratios of increases in the heavy element opacity. To augment this opacity we employed the simple expedient of multiplying the quantity $Z$ by a factor of 5 in the two places in which it appears in the formula. It is to be emphasized that this change was made only in the opacity subroutine; elsewhere in the program, $Z$ was left alone so that the equation of state suffered no altera- 
TABLE 1

LNA Results for Double-Mode CePheId Model ${ }^{\mathrm{a}}$

\begin{tabular}{ccccc}
\hline \hline Model & $P_{0}$ & $\left(-\sigma_{i} / \sigma_{r}\right)_{0}$ & $(\Delta \phi)_{0}$ & $P_{1} / P_{0}$ \\
\hline NO $\ldots \ldots . .$. & 3.126 & $8.92(-4)$ & 122 & 0.742 \\
AMO $\ldots .$. & 3.306 & $6.47(-4)$ & 117 & 0.713 \\
\hline
\end{tabular}
0.02 .

${ }^{\mathrm{a}} M=5 M_{\odot} ; L=1100 L_{\odot} ; T_{e}=5800 \mathrm{~K} ; X=0.70 ; Z=$

tions. Due to the nonlinear nature of the Stellingwerf formula, it turned out that the effect of our scheme was to increase metal opacities by factors of 2-3 (rather than 5 ) in the temperature range between $10^{5}$ and $10^{6} \mathrm{~K}$. The increase of total opacity in these layers was by a factor of 1.5 to 2 . Opacity changes in the hydrogen and helium ionization zones were negligible.

Table 1 compares linear nonadiabatic (LNA) results of normal-opacity (NO) and augmented-metals-opacity (AMO) calculations, for an evolutionary mass model in the double-mode Cepheid regime. The first entries are period, growth rate, and phase shift (maximum light minus minimum radius, in degrees) for the fundamental mode. The last entry is the ratio of first overtone to fundamental period. Table 2 compares NO and AMO results for a bump Cepheid model. Here the last entry is the ratio of second overtone to fundamental period.

From the last columns of Tables 1 and 2, one notes that the period-ratio discrepancy has disappeared in the AMO models. Furthermore, these models yield growth rates and, particularly, phase shifts which have not changed drastically from their NO equivalents. The latter result removes an objection raised by Fricke, Stobie, and Strittmatter (1971), who found that certain opacity changes altered the phase shifts unacceptably.

Table 3 presents calculations for the RR Lyrae regime. The first two rows display results for NO and AMO models respectively. One notes that the period ratio $P_{1} / P_{0}$ changes negligibly, so that the AMO, as well as the NO, is in agreement with observations. The lack of alteration in $P_{1} / P_{0}$ is, of course, due to the fact that these are Population II models with low $Z$. In the last row we present results for an augmented total opacity (ATO) model, in which the entire opacity has been multiplied by a factor of 2.5 at all levels hotter than $10^{5}$ $\mathrm{K}$. The period ratio $P_{1} / P_{0}$ is now far too small. This result demonstrates that increases in the hydrogen and helium opacities are not acceptable. Only AMO models will remedy the period-ratio problems in the Cepheid regime while leaving the (correct) RR Lyrae periods alone.

\section{THE $\beta$ CEPHEI STARS}

The long-standing problem of the $\beta$ Cephei variables has been reviewed by Cox (1976). The energizing mechanism for pulsations in these objects remains an unsolved
TABLE 2

LNA RESULTS FOR BUMP CEPHEID MODEL

\begin{tabular}{ccccc}
\hline \hline Model & $P_{0}$ & $\left(-\sigma_{i} / \sigma_{r}\right)_{0}$ & $(\Delta \phi)_{0}$ & $P_{2} / P_{0}$ \\
\hline NO $\ldots \ldots . .$. & 9.731 & $4.10(-3)$ & 113 & 0.539 \\
AMO $\ldots .$. & 10.58 & $2.14(-3)$ & 106 & 0.498 \\
\hline
\end{tabular}

${ }^{\mathrm{a}} M=7 M_{\odot} ; L=4742 L_{\odot} ; T_{e}=5623 \mathrm{~K} ; X=0.70 ; Z=$ 0.03

TABLE 3

LNA RESULTS FOR RR LYRAE MODEL

\begin{tabular}{ccrcc}
\hline \hline Model & $P_{0}$ & $\left(-\sigma_{i} / \sigma_{r}\right)_{0}$ & $(\Delta \phi)_{0}$ & $P_{1} / P_{0}$ \\
\hline NO $\ldots \ldots . .$. & 0.541 & $5.82(-4)$ & 79 & 0.744 \\
AMO $\ldots . .$. & 0.544 & $4.88(-4)$ & 77 & 0.742 \\
ATO $\ldots . .$. & 0.564 & $-9.47(-5)$ & 78 & 0.715 \\
\hline
\end{tabular}

${ }^{\mathrm{a}} M=0.65 M_{\odot} ; L=60.3 L_{\odot} ; T_{e}=7000 \mathrm{~K} ; X=0.70 ; Z=$ 0.001 .

question. Although many solutions have been put forward, none are generally accepted. Stellingwerf (1978) proposed that driving in these stars may be provided by an opacity feature due to the $\mathrm{He}^{+}$ionization edge near $T=1.5 \times 10^{5} \mathrm{~K}$. While the destablizing effect of this feature (a slight bump in the run of opacity with temperature) is not enough to excite $\beta$ Cephei pulsations, it was shown by Stellingwerf that an artificial increase of about $70 \%$ in the opacity near $\log T=5.2$ would suffice to make the models unstable. Furthermore, it was argued that the instability strip produced by such models satisfied the most important observational constraints.

Following a suggestion by Stellingwerf (private communication), we have plotted for one of our classical Cepheid models $\left(M=7 M_{\odot}, L=4742 L_{\odot}, T_{e}=5623\right.$ $\mathrm{K})$ the run of opacity with temperature for both the NO and AMO cases. These plots are shown in Figure 1. The NO model shows a slight bump in the vicinity of $\log T$ $=5.2$; in the AMO model, this feature is enhanced considerably. The picture is very similar to that displayed in Figure 5 of Stellingwerf (1978). Although the present classical Cepheid models differ in density from models in the $\beta$ Cephei regime, it is nonetheless expected that AMO models of the latter objects would also show the enhanced opacity feature. It is thus quite possible that, by the single stroke of augmenting the heavy element opacities by factors of $2-3$, we can bring into line with the theory of stellar structure and evolution not only the double-mode and bump Cepheids, but the $\beta$ Cephei pulsators as well.

\section{DISCUSSION}

An early, detailed description of the Los Alamos opacities was published by Cox (1965). For the physical 


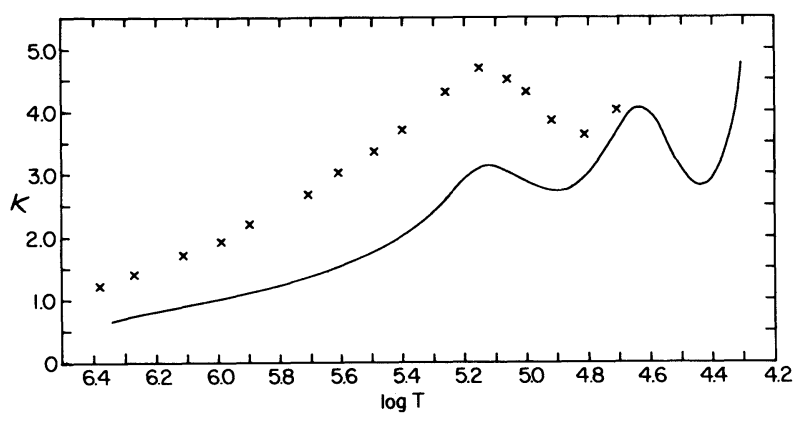

FIG. 1. - Run of opacity vs. temperature for the model $M=7$ $M_{\odot}, L=4742 L_{\odot}, T_{e}=5632 \mathrm{~K}$. Solid Curve, NO; crosses, the AMO model where it departs from the NO model.

regime of present interest, Cox indicates that heavy ions were treated in an essentially hydrogenic approximation, including electron screening. In subsequent years, a number of improvements and refinements were made in the Los Alamos code, some of which are discussed briefly by Cox and Tabor (1976), and in more detail by Huebner (1982). The former authors dismiss the Carson opacities, quoting criticism of the Thomas-Fermi method (e.g., Cloutman 1973), but critiques of the hydrogenic approximation (e.g., Carson and Hollingsworth 1968) are also left standing. Cox and Tabor further reject the idea of Watson $(1969,1970)$ that detailed allowance for autoionization lines could augment the opacities significantly. Huebner (1982) suggests that possibly Watson counted the absorption from some lines twice.

The treatment of heavy ions is extremely complex. Concerning photoionization, there are virtually no experimental data; for bound-bound transitions in these ions, including the vacuum ultraviolet (i.e., the range of our interest), the situation is equally bad (Samson 1982). Thus we are forced to rely exclusively on calculated energy levels and transition probabilities.

The theory of atomic photoionization is reviewed by Starace (1982). This theory may be directly linked to experiment only in the case of neutral atoms where a limited number of precise laboratory data are available. Comparison of theory with experiment in a handful of cases indicates that central potential models tend to overestimate cross sections near threshold and underestimate them at higher energies. This also applies to hydrogenic models, as shown by Missavage, Manson, and Daum (1977). More sophisticated calculations which attempt to include directly electron correlations may disagree among themselves in difficult cases by factors as large as 2 (Starace 1982).

Reilman and Manson (1979) argue that central potential models ought to be more accurate for ions than for neutral atoms. Indeed, for higher states of ionization, cross sections calculated by different methods seem to agree within narrower limits (e.g., Missavage, Manson, and Daum 1977). Reilman and Manson quote errors of $20 \%-200 \%$ for neutral atoms and $10 \%$ for highly charged ions. (However, see Starace 1982 for at least one case in which a central potential calculation was in error by a factor of 5.) These estimates do not, however, apply where autoionization is present.

Turning now to the case of bound-bound transitions in heavy ions, the picture seems even more cloudy. The National Bureau of Standards compendium of atomic transition probabilities (Wiese, Smith, and Glennon 1966; Wiese, Smith, and Miles 1969) shows very few entries at wavelengths in the vacuum ultraviolet. Furthermore, in those cases for which cross sections have been calculated, the uncertainties are usually described as "larger than 50\%," i.e., as open-ended. Once more, there is no direct link with experiment.

It is against this background that we make the assertion that heavy element opacities in the region between $10^{5} \mathrm{~K}$ and $2 \times 10^{6} \mathrm{~K}$ may plausibly have been underestimated by a factor of $2-3$. While there is no evidence that published transition probabilities are preponderantly on the low side of their actual values, it seems reasonable to maintain that opacities due to metals are more likely to rise than to fall with more extensive calculations. This is because the inclusion of any additional source of opacity will almost certainly result in an augmentation of the total. Such an effect has been strikingly illustrated by Magee, Merts, and Huebner (1975), who showed that the inclusion of a number of narrow lines in the Los Alamos code increased the opacity by as much as $40 \%$ in the regime $10<k T<200$ $\mathrm{eV}$, i.e., exactly where we have proposed an increase. Clearly, if this result is typical, it would not take the addition of too many new lines before the required rise is realized.

Unfortunately, we feel unable at present to advance more specific suggestions as to how the opacities might be increased. The dissemination by the Los Alamos group (or any other group) of an opacity atlas listing the major opacity sources at each wavelength and indicating in some way how the relevant transitions were treated would constitute an enormous step forward in opening up this important area to the astrophysical community at large. Further progress might then be made not only by astronomers interested in the finished product, but also by atomic physicists who would then have an incentive for calculations that otherwise might never be performed. Ionic opacities affect virtually all domains in astrophysics. It is strongly urged that new initiatives be undertaken soon in this vital field.

We are happy to acknowledge very helpful discussions with A. N. Cox, J. A. R. Samson, and A. F. Starace. This work was supported by the National Science Foundation under grant AST 8105064. 
Carson, T. R., and Hollingsworth, H. M. 1968, M.N.R.A.S., 141, 77.

Carson, T. R., and Stothers, R. 1976, Ap. J., 204, 461.

Cloutman, L. D. 1973, Ap. J., 184, 675.

Cox, A. N. 1965, in Stars and Stellar Systems, Vol. 8, Stellar Structure, ed. L. H. Aller and D. B. McLaughlin (Chicago: University of Chicago Press). 1980, Ann. Rev. Astr. Ap., 18, 15

Cox, A. N., Hodson, S. W., and Clancy, S. P. 1982, Ap. J., submitted.

Cox, A. N., and Tabor, J. E. 1976, Ap. J. Suppl., 31, 271.

Cox, J. P. 1976, in Proceedings of the Solar and Stellar Pulsation Conference, ed. A. N. Cox and R. G. Deupree (Los Alamos Scientific Laboratory: LA6544c), p. 127.

Davis, C. G., and Simon, N. R. 1981, Bull. AAS, 13, 522.

Fricke, K., Stobie, R. S., and Strittmatter, P. A. 1971, M.N.R.A.S., $154,23$.

Huebner, W. F. 1982, preprint

Jerzykiewicz, M., and Wenzel, W. 1977, Acta Astr., 27, 35.

Magee, N. H., Merts, A. L., and Huebner, W. F. 1975, Ap. J., 196, 617.

Missavage, D. W., Manson, S. T., and Daum, G. R. 1977, Phys. Rev. A, 15, 1001 .
Petersen, J. O. 1974, Astr. Ap., 34, 309.

Reilman, R. F., and Manson, S. T. 1979, Ap. J. Suppl., 40, 815.

Samson, J. A. R. 1982, Handbuch der Physik, Vol. 31, ed. W. Mehlhorn (Berlin: Springer-Verlag), in press.

Simon, N. R. 1981, Bull. AAS, 13, 871.

Simon, N. R., and Davis, C. G. 1982, Ap. J., submitted.

Simon, N. R., Lee, A. S., and Teays, T. J. 1980, Bull. AAS, 12, 862.

Starace, A. F. 1982, in Handbuch der Physik, Vol. 31, ed. W. Mehlhorn (Berlin: Springer-Verlag), in press.

Stellingwerf, R. F. 1975, Ap. J., 195, 441.

. 1978, A.J., 83, 1184.

Vemury, S. K., and Stothers, R. 1978, Ap. J., 225, 939.

Watson, W. D. $1969, A p . J ., 157,375$ Ap. J. Suppl., 19, 235.

Wiese, W. L., Smith, M. W., and Glennon, B. M., ed. 1966, Atomic Transition Probabilities, Vol. 1 (Washington, D.C.: National Bureau of Standards) (NSRDS-NBS 4).

Wiese, W. L., Smith, M. W., and Miles, B. M., ed. 1969, Atomic Transition Probabilities, Vol. 2 (Washington, D.C.: National Bureau of Standards) (NSRDS-NBS 22).

NoRman R. SimON: Behlen Laboratory of Physics, University of Nebraska, Lincoln, NE 68588-0111 